\title{
Mitigating Irreversible Capacity Losses from Carbon Agents via Surface Modification
}

\author{
Daniela Molina Piper ${ }^{\mathrm{a}, \mathrm{b}}$, Seoung-Bum Son ${ }^{\mathrm{b}}$, Jonathan J. Travis ${ }^{\mathrm{c}}$, Younghee Lee ${ }^{\mathrm{c}}$, \\ Sang Sub Han ${ }^{\mathrm{d}}$, Seul Cham Kim ${ }^{\mathrm{d}}$, Kyu Hwan Oh ${ }^{\mathrm{d}}$, Steven M. George ${ }^{\mathrm{a}, \mathrm{c}}$, Se-Hee Lee ${ }^{\mathrm{a}}$, \\ and Chunmei $\operatorname{Ban}^{\mathrm{b}, *}$ \\ ${ }^{a}$ Department of Mechanical Engineering, University of Colorado at Boulder, Boulder, \\ CO 80309 \\ b National Renewable Energy Laboratory, 1617 Cole Boulevard, Golden, C0 80401 \\ ${ }^{\mathrm{c}}$ Department of Chemistry and Biochemistry, University of Colorado at Boulder, \\ Boulder, CO 80309 \\ ${ }^{\mathrm{d}}$ Department of Material Science and Engineering, Seoul National University, Seoul,
} Korea 151-742

* Corresponding author: Dr. Chunmei Ban

E-mail: chunmei.ban@nrel.gov, Tel.: 303-384-6504, Fax: 303-384-6490 


\begin{abstract}
Greatly improved cycling performance has been demonstrated with conformally coated lithium-ion electrodes by atomic layer deposition (ALD) and molecular layer deposition (MLD) techniques. This paper reports the impact of coating on the electrode additives towards mitigating undesired parasitic reactions during cycling. The ALD and MLD coatings with conformality and atomic scale thickness control effectively stabilize the surface of the electrode components, and the current collector, resulting in the increase of coulombic efficiency throughout cycling. The organic fragment integrated into the recently developed MLD process allows the coating to possess excellent mechanical properties and enhanced ionic conductivity, which significantly reduces cell polarizations throughout cycling. This work validates the importance of ALD and MLD as surface modifiers and further demonstrates their versatility and compatibility with lithium-ion battery technology.
\end{abstract}

Keywords: Lithium-ion battery, Atomic layer deposition, Molecular layer deposition, Irreversible capacity loss. 


\section{Introduction}

The high electrical conductivity and chemical inertness of carbon black (CB) have led to its widespread use as a conducting additive [1-6] for manufacturing positive and negative electrodes in lithium-ion batteries. It is generally accepted that the role of the conducting agent is to pave the electrical pathways to electronically connect the electrochemically active particles throughout the laminated electrodes. Nevertheless, the negative impact of the conducting agent on the electrode performance has been continuously overlooked.

Previous works have shown that the irreversible capacity of both cathodes and anodes strongly depend on the content of $\mathrm{CB}$ in the composite electrodes. [6-9] There have even been several reports on $\mathrm{CB}$ as a potential anode material for lithium-ion batteries. [10-14] However, it has been found to give a relatively low charge-discharge capacity and a large first-cycle irreversible capacity, due to the intrinsic properties of the CB material. Due to the high surface area compared to the active materials, electrolyte reduction on the $\mathrm{CB}$ is likely to contribute substantially to the irreversible capacity through the formation of a solid electrolyte interphase (SEI) layer. Thereby, it is essential to study the effects of all the electrode components individually, and potentially attempt to mitigate the negative outcomes from each component. In this study, we focus on the irreversible capacity losses attributed to $\mathrm{CB}$ conductive agents and their mitigation via surface coating modifications.

Since irreversible reactions occurring on the electrode surface lead to capacity losses, an intuitive prevention method would be to modify the surface using a passivating layer. As demonstrated in previous work, ALD and MLD have been successfully implemented to passivate the liquid electrolyte/electrode interface from irreversible capacity losses. [15-21] The aforementioned works used ALD [15-20] to coat cathode and anode composite electrodes, and MLD [21] to coat high capacity anode composite 
electrodes, successfully improving the performance of both. These relatively new strategies have been continuously proven to be highly effective in enhancing the electrochemical performance of lithium-ion electrodes. Inspired by the results from such works, we focused on using ALD and MLD surface modification on electrodes containing only $\mathrm{CB}$ and polyvinylidene difluoride (PVDF) binder enabling a true identification and mitigation of the irreversible capacity losses attributed to the $\mathrm{CB}$ conductive additives, further corroborating the importance of surface coating via ALD and MLD. 


\section{Experimental}

\subsection{Material preparation}

Material Preparation of the CB-PVDF based composite electrodes was arranged by spreading CB powder $\left(75 \mathrm{~m}^{2} \mathrm{~g}^{-1}\right.$ surface area, Alpha Aesar), and PVDF mixed in $\mathrm{N}$ methyl pyrolidinone solvent (1:1 wt. ratio) on a piece of $\mathrm{Cu}$ foil. Once the electrodes were dried and calendared some were treated with ALD and others with MLD coating. Before assembling the cells the $1 / 2$ " punched electrodes were dried overnight $\left(120^{\circ} \mathrm{C}\right)$ in a vacuum oven. Cells were assembled in an Ar-filled glove box and tested at room temperature. Electrochemical measurements were all normalized based on the mass of CB in each electrode.

\subsection{Surface coating application}

ALD $\mathrm{Al}_{2} \mathrm{O}_{3}$ (AO) films were grown directly on the CB-PVDF composite electrodes using a pancake reactor. [19] The typical growth rate for the AO chemistry in this static reactor is $\sim 2 \AA$ per cycle. AO ALD has a typical growth rate of $1 \AA$ per cycle in traditional viscous flow reactors. It is higher in a static reactor due to imperfect purging and higher surface area materials. The $\mathrm{AO}$ reaction sequence was: i) trimethylaluminum (TMA) dose to 1.0 Torr; ii) TMA reaction time; iii) evacuation of reaction products and excess TMA; iv) $\mathrm{N}_{2}$ dose to 20.0 Torr ; v) $\mathrm{N}_{2}$ static time ; vi) evacuation of $\mathrm{N}_{2}$ and any entrained gases; vii) $\mathrm{H}_{2} \mathrm{O}$ dose to 1.0 Torr, viii) $\mathrm{H}_{2} \mathrm{O}$ reaction time; ix) evacuation of reaction products and excess $\mathrm{H}_{2} \mathrm{O} ; \mathrm{x}$ ) dose $\mathrm{N}_{2}$; xi) $\mathrm{N}_{2}$ static time; and xii) evacuation of $\mathrm{N}_{2}$ and any entrained gases. Steps iv, v, and vi were repeated 5 times between TMA and $\mathrm{H}_{2} \mathrm{O}$ doses. This sequence constitutes of one cycle of ALD AO. The electrodes were coated with 20 cycles of AO ALD and the reaction was conducted at $180{ }^{\circ} \mathrm{C}$.

MLD aluminium alkoxide, inorganic-organic films were also grown directly on 
CB-PVDF composite electrodes using a pancake reactor. [19] The typical growth rate for

the particular inorganic-organic chemistry used in this work is of $7.5 \AA$ per cycle. The inorganic-organic reaction sequence was: i) Dose inorganic-metal for 2 seconds, ii) hold inorganic-metal pressure static for 90 seconds, iii) flow purge for 180 seconds, iv) 5 cycles of argon static purge, v) dose organic molecule for 2 seconds, vi) hold organic pressure static for 120 seconds, vii) flow purge for 240 seconds, viii) 7 cycles of argon static purge. Flow purge is performed by pumping out excess precursors and reaction byproducts while flowing argon through the reactor. Argon static purge is performed by dosing argon for 20 seconds, holding argon pressure static for 5 seconds, pumping for 45 seconds, and flow purging for 20 seconds. This sequence constitutes of one cycle of MLD inorganic-organic film. The electrodes were coated with 10 cycles of inorganicorganic MLD and the reaction was conducted at $140{ }^{\circ} \mathrm{C}$.

\subsection{Electrochemical measurements}

Electrochemical measurements were carried out using an $\operatorname{Arbin}^{\mathrm{TM}} 2000$ battery test station and a Biologic battery test station. All cells were assembled in an Ar-filled glove box using the as-prepared bare, ALD coated, and MLD coated CB-PVDF electrodes as the working electrodes and lithium metal foil as the counter electrode. The electrolyte was $1 \mathrm{M} \mathrm{LiPF}_{6}$ dissolved in a 1:1 (volume ratio) mixture of ethylene carbonate (EC) and diethyl carbonate (DEC), the separator was a glass micro-fiber disk (Whatman ${ }^{\mathrm{TM}} \mathrm{GF} / \mathrm{F}$ ) and the shell was a stainless steel CR2032 coin cell (Pred. Materials). We used a constant current constant voltage (CCCV) testing scheme to cycle the cells and used a potential range of $0.05-1.5 \mathrm{~V}$ vs. $\mathrm{Li} / \mathrm{Li}^{+}$. The cells were discharged (lithiated) and charged (delithiated) with a current of $50 \mu \mathrm{A} \mathrm{mg}^{-1}$ of $\mathrm{CB}$ for the first 10 cycles and a current of $100 \mu \mathrm{A} \mathrm{mg}{ }^{-1}$ of $\mathrm{CB}$ for subsequent cycling between various voltage ranges including that of an anode and a cathode. 
Electrochemical impedance spectroscopy (EIS) was performed using the Biologic VMP3. The AC impedance measurements were recorded using a signal with an amplitude of $5 \mathrm{mV}$ and a frequency from $1 \mathrm{MHz}$ to $10 \mathrm{mHz}$. EIS were conducted for different electrochemical cycles, especially focused in the fully lithiated state of charge $\left(0.05 \mathrm{~V}\right.$ vs. $\left.\mathrm{Li} / \mathrm{Li}^{+}\right)$. To further study the redox reactions occurring at higher potentials than those utilized for the electrochemical measurements $\left(0.05-1.5 \mathrm{~V}\right.$ vs. $\left.\mathrm{Li} / \mathrm{Li}^{+}\right)$, cyclic voltammetry was applied to both CB-PVDF electrodes containing a copper $(\mathrm{Cu})$ current collector and aluminum (Al) current collector in representation of an anode and cathode electrode, respectively. Cyclic voltammetry was performed using a potential range of 2 4.5 V for the CB-PVDF electrode using the $\mathrm{Cu}$ current collector, and $2-5 \mathrm{~V}$ for $\mathrm{CB}$ PVDF electrode using the Al current collector and both of these were conducted at a scan rate of $10 \mathrm{mV} \mathrm{sec}^{-1}$.

\subsection{Physical characterization}

Material Characterization was done with the use of a focused ion beam, FIB (FEI, NOVA200 dual beam system), equipped with and air-lock chamber to prepare the transmission electron microscopy (TEM) samples. $\mathrm{Ga}^{+}$ion source is used for FIB sectioning. The use of an air-lock system enables the observation of lithiated CB-PVDF electrodes without any exposure to air. Air exposure on lithiated sample causes drastic structural changes due to oxidation. However, this mobile air-lock system maintains a vacuum state while samples are loaded from the glove box to the FIB chamber. [22]

TEM analysis was performed with a FEI Tecnai F20 operated at 200keV. TEM samples are prepared using our FIB air-lock system, descripted in the previous work [23]. A typical TEM sample preparation sequence follows: (1) Pt deposition for the protection of the desired surface area. (2) Cross-sectioning of the electrode with Ga+ source. (3) 
using a manipulating probe (100.7TM, Omniprobe). (4) Further sample thinning with a beam condition of $10 \mathrm{kV}$ and $30-50 \mathrm{pA}$ is performed until a $50-90 \mathrm{~nm}$ thickness is achieved (optimized conditions for TEM observation). 


\section{Results and Discussion}

TEM and Fast Fourier Transform (FFT) were utilized to characterize the microstructure of the CB-PVDF electrodes employed in this work. Figure 1 presents TEM images (Fig. 1a-d) and FFT (inset in Fig. 1d) of an uncycled bare CB-PVDF electrode and TEM images (Fig. 1e and 1f) and FFT (inset in Fig. 1f) of a fully lithiated bare CB-PVDF electrode. The images show the pristine $\mathrm{CB}$ particles having an average primary particle size of $50 \mathrm{~nm}$ that readily fuses to form aggregates as big as $500 \mathrm{~nm}$. However, the high-resolution TEM images indicate that the amorphous particle is composed of very small crystallites. In a microstructural model proposed by Riley, the CB particle was depicted as a random distribution of small crystallites with a size of mosaic blocks calculated to be $2 \mathrm{~nm}$. [24] As shown in the high-resolution TEM, the CB powder includes nanometric aggregates of fused sub-nanometric particles, which closely relates with the suggested model. Amorphous carbon FFT patterns are observed for both uncycled and lithiated CB-PVDF electrodes during the initial lithiation. With relatively small volume changes, the lithiated CB-PVDF electrode maintains the original structure without drastic mechanical deficiencies. However, we do observe surface alterations on the CB particles after the electrode is fully lithiated, as shown in Fig. 1e and 1f. The observed alterations are attributed to the reduction reactions and SEI formation occurring upon lithiation of the $\mathrm{CB}$ particles.

The observed surface effects have been previously characterized with different methods and have been labeled as major irreversible redox reactions. A clear correlation has been shown between irreversible capacity and CB content, with a substantial increase in irreversible capacity with increasing amount of the high surface area of CB. [6] Xing and Dahn showed that the irreversible capacity is not only a measure of the amount of SEI, but can also relate to the reduction of surface species on the carbon agents. [25] 
Many chemically active groups are likely to be present on the high surface area of carbon agents, and the reduction of these during the first cycle will contribute to the first-cycle irreversible capacity under SEI formation. Thus, the protection of electrode surfaces and mitigation of irreversible charge losses due to unwanted side reactions is of paramount importance. TEM micrographs of AO ALD coated CB particles are depicted in Figure S1 showing a conformal and complete coating throughout the high surface area of the particles. The significance of the CB particles' surface protection will be further substantiated by various electrochemical experiments.

Electrochemical characterization was utilized to identify the reversibility of electrochemical reactions in the carbon additives. The bare CB-PVDF electrode shows a much higher specific charge capacity than both coated electrodes (Fig. 2a). This behavior is consistent with previous work done with ALD and MLD surface modifications, which attributed this effect to be a dependence on the coating thickness. [15, 21, 26] For this work, we have employed coatings on the thicker side of the spectrum for electrode surface coatings ( 4 $\mathrm{nm}$ for ALD and $\sim 7.5 \mathrm{~nm}$ for MLD), which explains the lower capacities observed in the electrochemical studies. Nevertheless, all of the electrodes show a very good cycling stability attesting to the coatings' abilities to maintain the cell performance. Figure $\mathbf{2 b}$ presents the coulombic efficiency $(\mathrm{CE})$ of the bare, ALD coated, and MLD coated electrodes. The first CE of all the electrodes stands around $30 \%$. This low initial $\mathrm{CE}$ is consistent with other work done on different conducting carbon materials, $[6,27,28]$ suggesting this poor initial efficiency is an intrinsic property of the amorphous carbon. Note that the CB-PVDF electrode shows a tremendous uptake of $\mathrm{Li}^{+}$, as well as capacity loss at the first cycle. This behavior suggests that a reduced amount of carbon agents during composite electrode manufacturing is needed in order to improve the first-cycle efficiency. After the first cycle, all carbon electrodes show stable cycling performance, although the bare CB-PVDF electrode shows about $0.7 \%$ irreversible loss 
per cycle. The utilization of surface coatings, particularly ALD, helps to mitigate these unceasing irreversible losses and maintains fully reversible cycling.

The first cycle voltage profile for all of the electrodes was analyzed to further study the ALD and MLD capabilities in altering the interfacial reactions between the CBPVDF electrodes and liquid electrolyte (Fig. 2c). The irreversible capacity quasi-plateau observed at $0.8 \mathrm{~V}$ during initial lithiation is very prominent in the bare electrode and is ascribed to the reduction of the liquid electrolyte towards SEI formation on the surface of the electrode. $[29,30]$ Interestingly though, this SEI voltage segment in both ALD and MLD coated samples was suppressed. However, despite the reduction of the SEI formation voltage segment in both coated electrodes, the sustainable cycling performance obtained in the coated electrodes implies that a stabilized electrode surface was still achieved by using ALD and MLD coatings. This stabilized coated surface observed suggests that ALD and MLD act as an artificial SEI. We believe that the ALD and MLD coating modifies the surface of the electrodes enhancing the reductive environment the electrolyte experiences, thus passivating the significant irreversible charge losses that come from the SEI formation observed on the bare CB-PVDF electrodes. Similar results were obtained when the same electrochemical tests were conducted at higher temperature $\left(55^{\circ} \mathrm{C}\right)$, indicating that the ALD and MLD coatings retain the ability to protect and stabilize the surface of the CB-PVDF electrodes even under harsh environments (Figure 3). The continuous protection from secondary surface reactions is vital to maintain desirable performance throughout battery progression. To further probe the protective properties of the nanoscale surface coatings, high-energy Si-based anodes were coated with both ALD and MLD and compared to an uncoated electrode. The first lithiation voltage profiles were analyzed (Figure S2) showing, once again, the mitigation of initial irreversible charge losses consistent with our CB-PVDF electrochemical results (Fig. 2c) and previous works. [15-21] These results validate the importance of ALD and MLD as 
surface modifiers. The passivation of surface redox reactions allows for better performance and mitigates the growth of electrode polarization.

Electrochemical impedance has been measured to investigate the polarization of CB-PVDF electrodes during cycling. Figure 4 presents the Nyquist plots and the fitting with a Voigt-type equivalent circuit composed of resistors (R), constant-phase elements (CPE) and Warburg impedance (W). The impedance data was collected for the bare, ALD coated, and MLD coated CB-PVDF electrodes during an uncycled state (Fig. 4a), full initial lithiation state of charge (Fig. 4b), and after the $40^{\text {th }}$ fully lithiated state of charge (Fig 4c). Initially, the ohmic resistance $\left(R_{s}\right)$ of the uncycled electrodes does not seem to be significantly affected by the addition of the ALD and MLD electrode surface coatings (inset in Fig. 4a). The slight increase of the initial internal resistance for the ALD coated CB-PVDF electrode compared to that of the bare electrode is attributed to the insulating properties of ALD AO. $[17,19,26]$ The MLD coated electrode has no $R_{s}$ variation from the bare electrode due to MLD's polymeric characteristics and its capabilities to soak up liquid electrolyte without being a major influence on the internal resistance of the cell. [21] The charge transfer resistance initially observed is lower for the coated samples, and this behavior could be due to the change in surface/electrolyte environment. Further studies are in progress to specifically pinpoint the elements involved in this initial charge transfer behavior.

Furthermore, the impedance of the bare and coated electrodes was studied at the $1^{\text {st }}$ lithiation cycle and $40^{\text {th }}$ lithiation cycle with the respective fitted data $(+)$ according to the equivalent circuit depicted (inset in Fig. 3c). After full lithiation of the bare CBPVDF electrodes we see the growth of SEI impedance (high frequency semicircle). The SEI resistance, $\mathrm{R}_{\mathrm{SEI}}$, was highest for the bare electrode in this first initial lithiation cycle. This higher $\mathrm{R}_{\mathrm{SEI}}$ seems to be directly related to the formation and growth of SEI layer, 
consistent with the initial voltage plateau observed in the bare CB-PVDF electrode. The coated electrodes show the formation of an $\mathrm{R}_{\mathrm{SEI}}$, which is expected, but with reduced polarization attributed to the passivating properties of the ALD and MLD coatings. The charge transfer resistances of all the electrodes, $\mathrm{R}_{\mathrm{ct}}$ (medium frequency semicircle), after full lithiation are lower than the pristine electrodes. This is explained by the activated surfaces of all the electrodes with $\mathrm{Li}^{+}$diffusion alleviating the charge transfer effect over the electrode/electrolyte interface. The SEI layer and insulating ALD coating passivate the surface to decrease interfacial activities. The lowest value observed in $\mathrm{R}_{\mathrm{ct}}$ comes from the MLD coated CB-PVDF electrode corroborating the polymeric capability to soak up liquid electrolyte significantly easing the charge transfer process.

The impedance resistances were analyzed again after the $40^{\text {th }}$ lithiated cycle (Fig. 4c). We observed that most of the $\mathrm{R}_{\mathrm{SEI}}$ decreased for all of the electrodes upon the stabilization of this natural surface layer formation throughout cycling progression, with the lowest resistance coming again from the MLD coated CB-PVDF electrode. On the other hand, we observed a drastic increment in the $\mathrm{R}_{\mathrm{ct}}$ for the bare electrode. The increase in the $\mathrm{R}_{\mathrm{ct}}$ as a function of cycle number is associated to material property changes occurring as a result of constant electrode/electrolyte interactions. [17] The surface of the bare CB-PVDF interface is not fully stable in the liquid electrolyte and appears to constantly be changing and increasing surface polarizations. CB is highly disordered with effectively a high density of edge-planes, so there are considerable sites available for continual interaction with the liquid electrolyte. [6] This degradation inhibits the charge transfer at the material surface. The ALD coated CB-PVDF also shows an increase in its $\mathrm{R}_{\mathrm{ct}}$, which is attributed again to the insulating properties of the thicker ALD film employed in this work. [17, 31] More importantly, after MLD coating, the $\mathrm{R}_{\mathrm{ct}}$ yields nearly no change as a function of cycle number when compared to the bare electrode. The steadier values of MLD $\mathrm{R}_{\mathrm{ct}}$ are ascribed to the added protection of the CB-PVDF 
electrode from the liquid electrolyte alleviating many unwanted electrode/electrolyte reactions.

To further substantiate the passivating properties of the ALD and MLD coatings, we analyzed their stability and protective properties against the liquid electrolyte for several cycles at a higher potential range of $2.0-4.5 \mathrm{~V}$ vs. $\mathrm{Li} / \mathrm{Li}^{+}$. Note that the high voltages were used here only for the stability evaluation of the $\mathrm{Cu}$ current collector, even though the voltage for the anode current collector is typically limited to below $3 \mathrm{~V}$. Cyclic voltammetry (CV) was conducted to probe this high potential region and observe the redox reactions occurring in the bare and coated electrodes. The electrodes were set up in an anode configuration using $\mathrm{Cu}$ foil as the current collector. Figure 5a depicts the $\mathrm{CV}$ redox responses for several cycles for the bare CB-PVDF electrode. As expected, two significant and continuous anodic and cathodic reactions were observed due to the oxidation (at $\sim 3.8 \mathrm{~V}$ ) and reduction (at $\sim 3.4 \mathrm{~V}$ ) of the $\mathrm{Cu}$ current collector, respectively. This redox behavior at this potential window for $\mathrm{Cu}$ correlates very well with other studies previously done. [31] Moreover, the same CV scheme was applied for the MLD coated (Fig. 5b) and the ALD coated (Fig. 5c) CB-PVDF electrodes. Both coated electrodes substantially mitigate the oxidation and reduction that occurs at higher voltage regions with the $\mathrm{Cu}$ current collector, especially with the use of ALD, which shows to almost completely prevent any reactions from occurring (Fig. 5c). The moderation of the $\mathrm{Cu}$ current collector's redox reactions observed with the use of ALD and MLD, demonstrates these coatings' capability to not only passivate the surface of the electrode film, but also fully protect all other elements of the cell that are likely to react and alter battery performance.

Considering how reactive $\mathrm{Cu}$ is at this high potential region and the possibility to completely camouflage any redox reactions coming from the electrode film, a CV of a 
bare and ALD coated CB-PVDF cathodes (Al current collector) was also investigated (Fig. 5d). The CV voltage range for the cathode electrodes analyzed was amplified to cover $2-5 \mathrm{~V}$ vs. $\mathrm{Li} / \mathrm{Li}^{+}$. As we projected, the bare CB-PVDF cathode electrode (black profile, Fig. 5d) did show to have a small irreversible reduction effect at $\sim 4.2 \mathrm{~V}$ throughout various cycles. We suspect this effect is directly related to the continuous electrolyte decomposition or oxidization on the high surface area of the CB-PVDF electrode film. [6] The mitigation of these constant irreversible charge losses is vital on the cathode electrode as well. With the use of ALD as a protective coating on the cathode electrode, we show the complete elimination of these unwanted secondary side reactions (blue profile, Fig. 5d). 


\section{Conclusion}

The complexity of the chemical and electrochemical reactions occurring in lithium-ion batteries has been demonstrated here by showing the influence that an element as simple as CB material can generate when used as a conductive additive in the anode and cathode composite electrodes. The irreversible charge losses observed from $\mathrm{CB}$ are too significant to be overlooked, especially when potential next generation lithium-ion battery materials, such as $\mathrm{S}$ and $\mathrm{Si}$, require the addition of higher conductive additive content due to their insulating trait. [32] The intrinsic properties of CB material and the negative effect it has, especially during initial cycling, makes it crucial to find ways to mitigate these unwanted charge losses or reduce its utilization as much as possible. In this work, we reiterate the irreversible effects that come from the utilization of $\mathrm{CB}$ conductive agents in composite electrodes and we offer a mitigation system via surface coating using ALD and MLD. The application of ALD and MLD conformal coatings as a surface modifier for $\mathrm{CB}$ conductive agents is established here to be a great option to alleviate many unwanted reactions occurring in the high surface area of $\mathrm{CB}$ material. ALD and MLD coatings have not only been proven to be an advancement for high capacity anodes $[21,33]$ and various cathodes, $[15-17,20]$ but also to be a protective system for other cell elements such as conductive agent materials and current collectors as demonstrated here. The versatility and compatibility of ALD and MLD surface modification makes these techniques a major innovation for lithium-ion battery technology. 


\section{Acknowledgements}

This work was supported by the Assistant Secretary for Energy Efficiency and Renewable Energy, Office of Vehicle Technologies for the U.S. Department of Energy under Contract No. DE-AC-36-08GO28308, subcontract No. NFT-8-88527-01 under the Batteries for Advanced Transportation Technologies (BATT) Program, and by a grant from the Fundamental R\&D Program for Technology of World Premier Materials funded by the Ministry of Knowledge Economy, Republic of Korea (10037919). We would also like to acknowledge the financial support from NREL Lab Directed Research and Development (LDRD) program. 


\section{References}

[1] K. Kinoshita, Carbon: Electrochemical and Physical Properties, Wiley, 1988.

[2] J. Lahaye, M.J. Wetterwald, J. Messiet, J. Appl. Electrochem., 14 (1984) 117-122.

[3] J. Lahaye, M.J. Wetterwald, J. Messiet, J. Appl. Electrochem., 14 (1984) 545-553.

[4] S. Mandal, J.M. Amarilla, J. Ibanez, J.M. Rojo, J. Electrochem. Soc., 148 (2001)

A24-A29.

[5] J.C. Biermann, M.J. Wetterwald, J. Messiet, J. Lahaye, Electrochim. Acta, 26 (1981) 1237-1239.

[6] L. Fransson, T. Eriksson, K. Edstrom, T. Gustafsson, J.O. Thomas, J. Power Sources, $101(2001) 1-9$.

[7] J.M. Tarascon, W.R. Mckinnon, F. Coowar, T.N. Bowmer, G. Amatucci, D. Guyomard, J. Electrochem. Soc., 141 (1994) 1421-1431.

[8] T. Takamura, M. Saito, A. Shimokawa, C. Nakahara, K. Sekine, S. Maeno, N. Kibayashi, J. Power Sources, 90 (2000) 45-51.

[9] F. Joho, B. Rykart, R. Imhof, P. Novak, M.E. Spahr, A. Monnier, J. Power Sources, 81 (1999) 243-247.

[10] G.B. Li, R.J. Xue, L.Q. Chen, Y.Z. Huang, J. Power Sources, 54 (1995) 271-275.

[11] K. Takei, N. Terada, K. Kumai, T. Iwahori, T. Uwai, T. Miura, J. Power Sources, 55 (1995) 191-195.

[12] A.K. Sleigh, U. Vonsacken, Solid State Ionics, 57 (1992) 99-102. 
[13] T.D. Tran, J.H. Feikert, X. Song, K. Kinoshita, J. Electrochem. Soc, 142 (1995) 3297-3302.

[14] R. Yazami, M. Deschamps, J. Power Sources, 54 (1995) 411-415.

[15] I.D. Scott, Y.S. Jung, A.S. Cavanagh, Y.F. An, A.C. Dillon, S.M. George, S.H. Lee, Nano Lett., 11 (2011) 414-418.

[16] Y.S. Jung, A.S. Cavanagh, L.A. Riley, S.H. Kang, A.C. Dillon, M.D. Groner, S.M. George, S.H. Lee, Adv. Mater., 22 (2010) 2172-+.

[17] L.A. Riley, S. Van Ana, A.S. Cavanagh, Y.F. Yan, S.M. George, P. Liu, A.C. Dillon, S.H. Lee, J. Power Sources, 196 (2011) 3317-3324.

[18] X.B. Meng, X.Q. Yang, X.L. Sun, Adv. Mater., 24 (2012) 3589-3615.

[19] Y.S. Jung, A.S. Cavanagh, A.C. Dillon, M.D. Groner, S.M. George, S.H. Lee, J Electrochem. Soc., 157 (2010) A75-A81.

[20] J.W. Kim, J.J. Travis, E. Hu, K.-W. Nam, S.C. Kim, C.S. Kang, J.H. Woo, X.-Q. Yang, S.M. George, K.H. Oh, S.-J. Cho, S.-H. Lee, J. Power Sources, 254 (2014) 190197.

[21] D. Molina Piper, M. Young, S.-B. Son, S.C. Kim, K.H. Oh, S.M. George, C. Ban, S.-H. Lee, Adv. Mater., 26 (2014) 1596-1601.

[22] S.B. Son, S.C. Kim, C.S. Kang, T.A. Yersak, Y.C. Kim, C.G. Lee, S.H. Moon, J.S. Cho, J.T. Moon, K.H. Oh, S.H. Lee, Adv. Energy. Mater, 2 (2012) 1226-1231. 
[23] S.B. Son, J.E. Trevey, H. Roh, S.H. Kim, K.B. Kim, J.S. Cho, J.T. Moon, C.M.

DeLuca, K.K. Maute, M.L. Dunn, H.N. Han, K.H. Oh, S.H. Lee, Adv. Energy. Mater, 1 (2011) 1199-1204.

[24] H.L. Riley, Chem. Ind., 58 (1939).

[25] W.B. Xing, J.R. Dahn, J. Electrochem. Soc, 144 (1997) 1195-1201.

[26] Y.S. Jung, A.S. Cavanagh, Y.F. Yan, S.M. George, A. Manthiram, J. Electrochem. Soc, 158 (2011) A1298-A1302.

[27] F. La Mantia, R.A. Huggins, Y. Cui, J. Appl. Electrochem., 43 (2013) 1-7.

[28] J.K. Hong, J.H. Lee, S.M. Oh, J. Power Sources, 111 (2002) 90-96.

[29] H.S. Kim, K.T. Kim, Y.S. Kim, S.W. Martin, Met. Mater-Int., 14 (2008) 105-109.

[30] K. Xu, Energies, 3 (2010) 135-154.

[31] M.C. Zhao, H.D. Dewald, F.R. Lemke, R.J. Staniewicz, J. Electrochem. Soc, 147 (2000) 3983-3988.

[32] N.-S. Choi, Z. Chen, S.A. Freunberger, X. Ji, Y.-K. Sun, K. Amine, G. Yushin, L.F. Nazar, J. Cho, P.G. Bruce, Angew. Chem. Int. Ed., 51 (2012) 9994-10024.

[33] L.A. Riley, A.S. Cavanagh, S.M. George, S.H. Lee, A.C. Dillon, Electrochem. Solid-State Lett., 14 (2011) A29-A31. 


\section{Figure Captions}

Figure 1. (a-c) TEM micrographs of uncycled bare CB-PVDF electrodes showing an average primary particle size of $50 \mathrm{~nm}$ that readily fuses to from $500 \mathrm{~nm}$ aggregates along with (d) TEM micrograph of a single particle at higher resolutions with its respective FFT pattern showing amorphous carbon. (e) TEM micrograph of fully lithiated bare CB-PVDF electrodes showing some surface alteration attributed to the reduction reactions occurring upon $\mathrm{Li}^{+}$insertion to the $\mathrm{CB}$ particles along with (f) TEM micrograph of a single particle at higher resolutions with its respective FFT pattern showing no phase changes during cycling (amorphous carbon pattern).

Figure 2. (a) Cyclic stability of bare (black profile), ALD coated (blue profile), and MLD coated (yellow profile) CB-PVDF electrodes along with their respective (b) coulombic efficiency. (d) Voltage profile of first lithiation and delithiation cycle of each bare, ALD coated and MLD coated CB-PVDF electrodes showing the reduction of irreversible charge losses for the coated electrodes.

Figure 3. (a) Cyclic stability of bare (red profile), ALD coated (cyan profile), and MLD coated (brown profile) CB-PVDF electrodes conducted at $55^{\circ} \mathrm{C}$ along with their respective (b) coulombic efficiencies. (d) Voltage profile of first lithiation and delithiation cycle of each bare, ALD coated and MLD coated CB-PVDF electrodes showing the reduction of irreversible charge losses for the coated electrodes even at high temperature testing $\left(55^{\circ} \mathrm{C}\right)$.

Figure 4. Nyquist plots resulting from EIS done on bare, ALD coated, and MLD coated CB-PVDF electrodes during an (a) uncycled state, (b) full initial lithiation state of charge, and (c) after the $40^{\text {th }}$ fully lithiated state of charge. Cycled electrodes were fitted 
according to the equivalent circuit depicted (inset in c) and represented by (+)

respectively.

Figure 5. (a) CV redox responses for several cycles for the bare CB-PVDF electrode, (b) MLD coated CB-PVDF electrode, (c) and the ALD coated CB-PVDF electrode showing significant reduction of $\mathrm{Cu}$ current collector redox reaction for the coated electrodes, especially with ALD. (d) CV of a bare CB-PVDF and ALD coated CB-PVDF cathodes (Al current collector) also showing the mitigation of continuous electrolyte decomposition or chemical reduction from other active species on the high surface area of the CB-PVDF electrode film. 
Figure 1

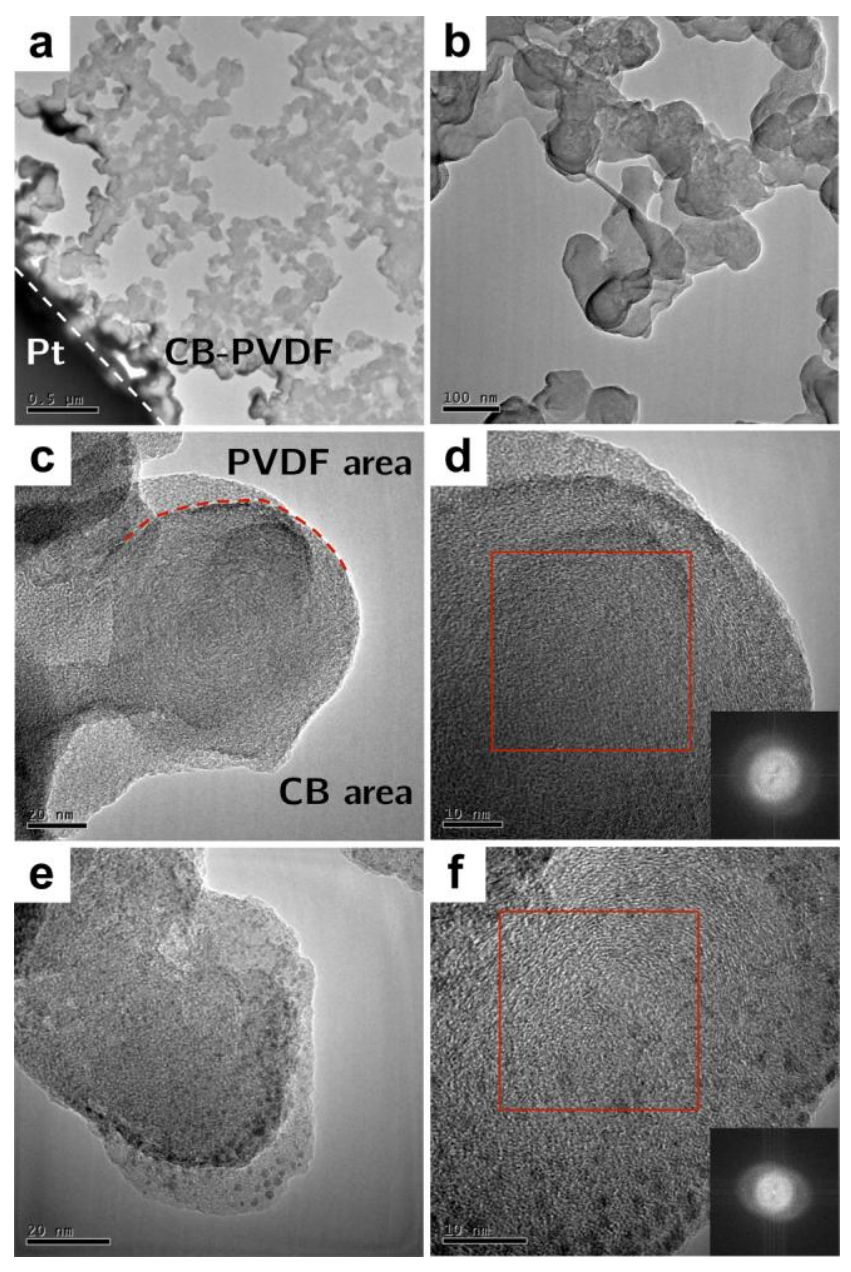


Figure 2
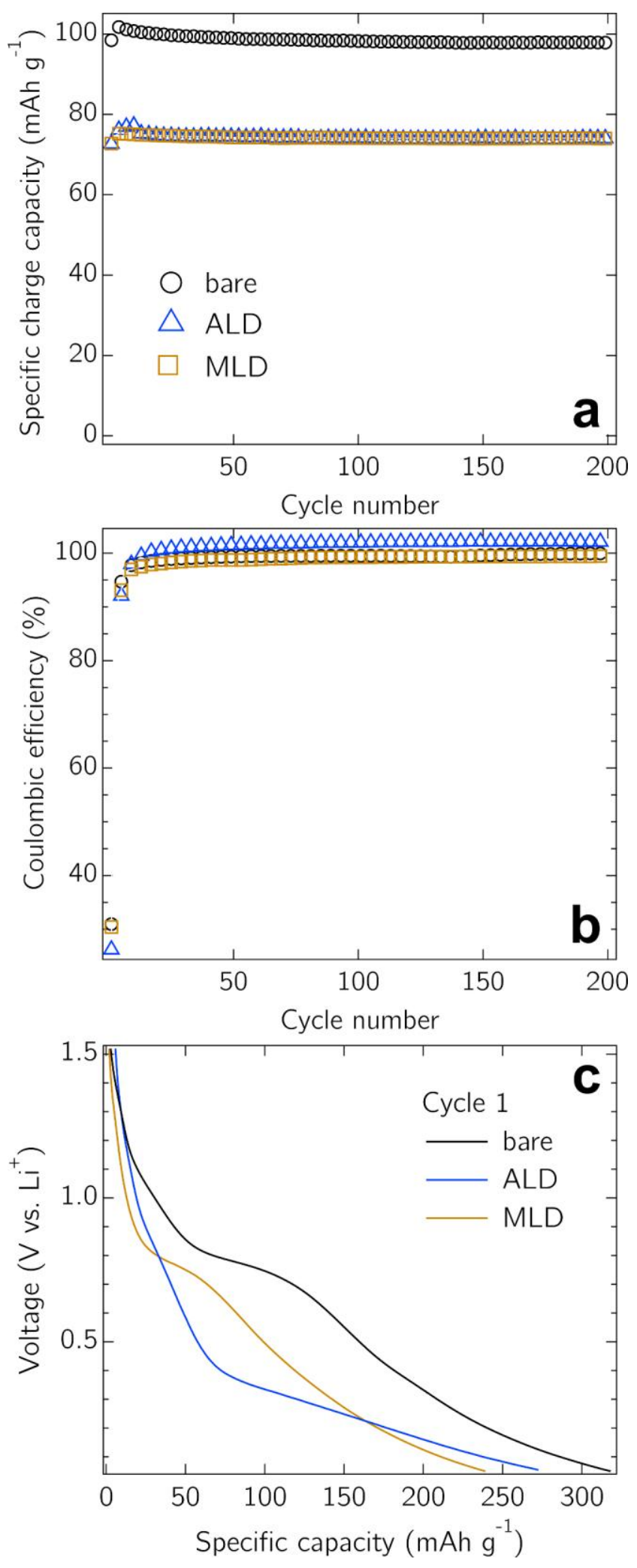
Figure 3
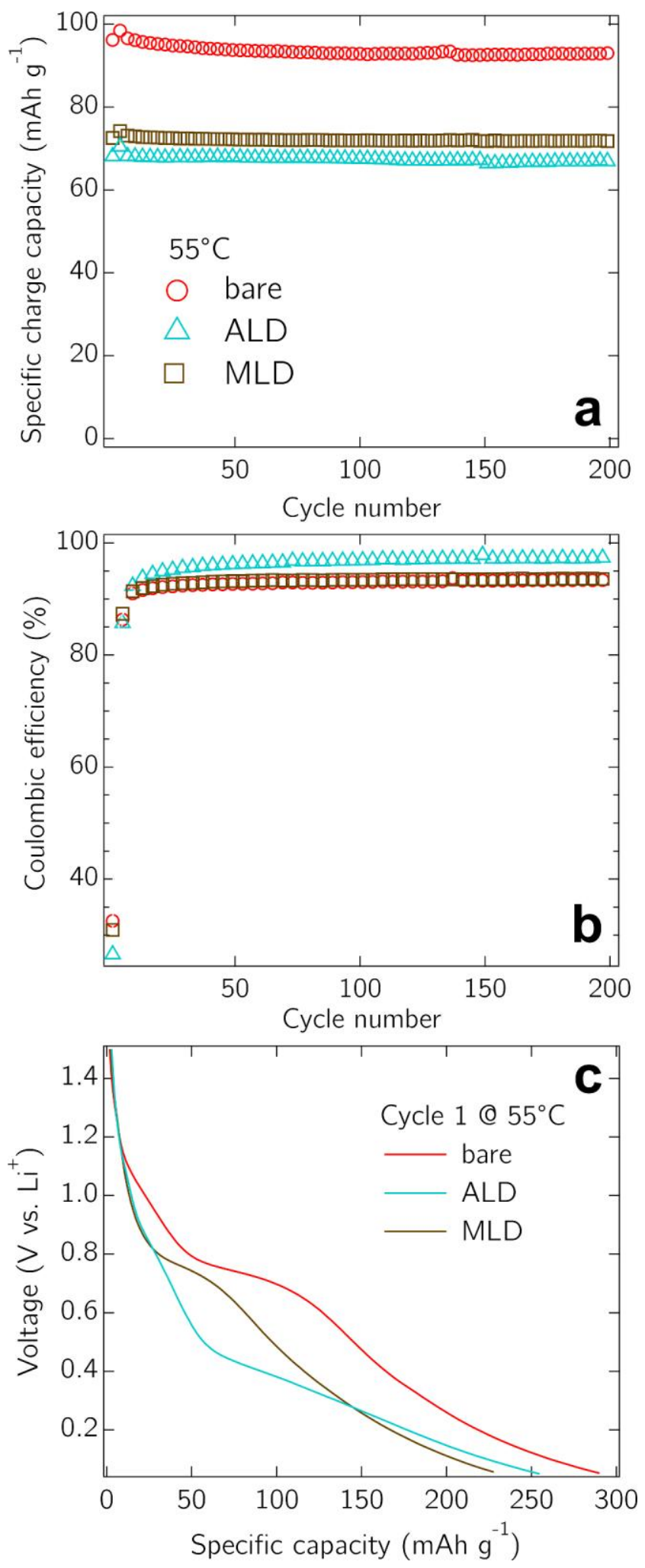
Figure 4
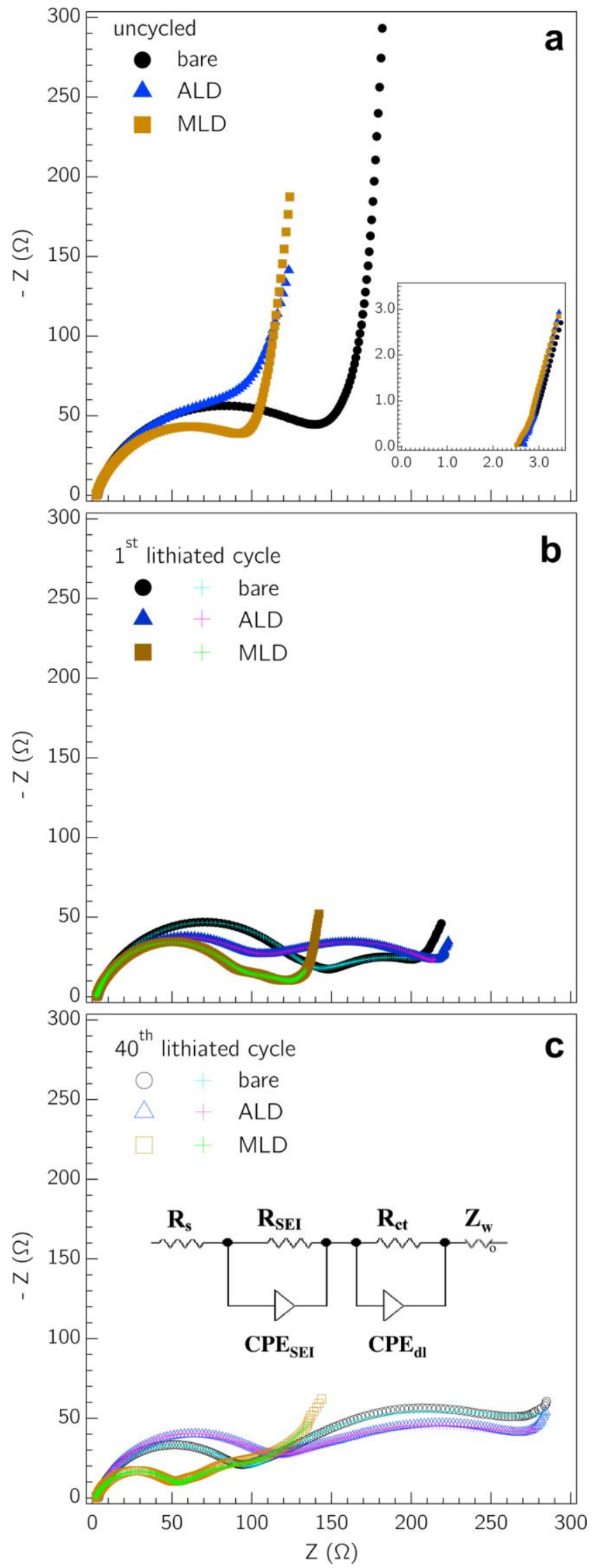
Figure 5
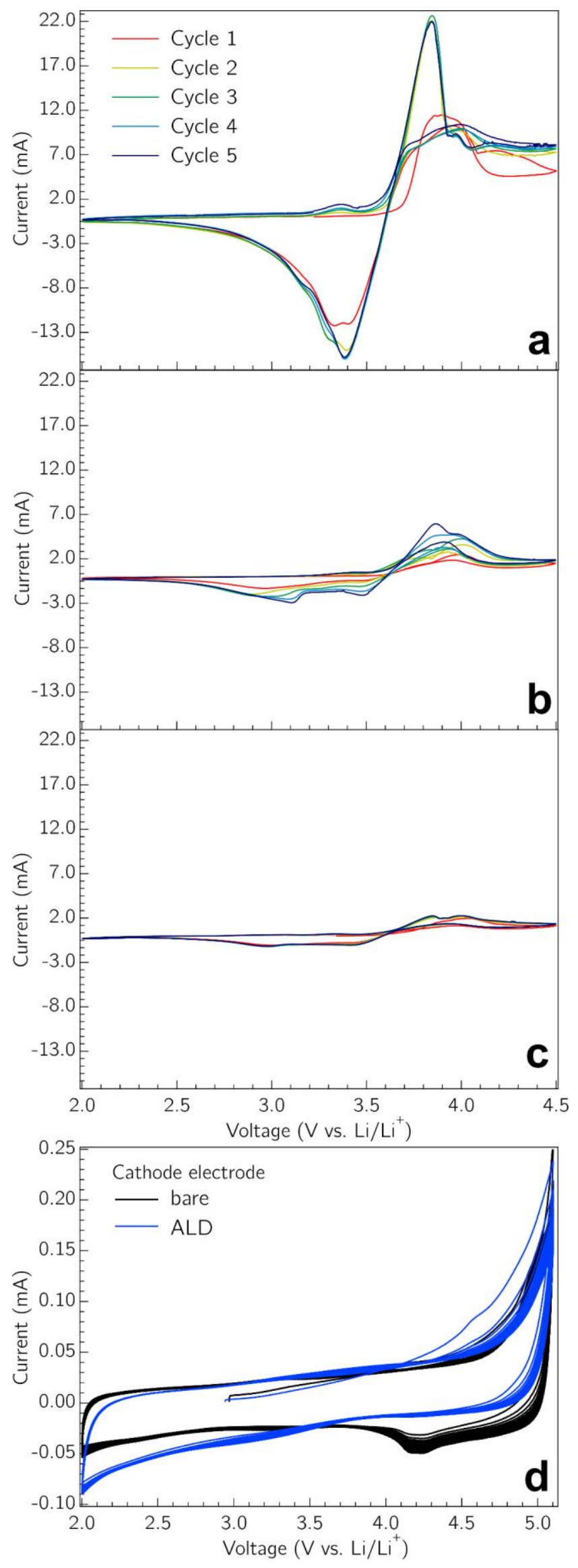